\title{
Influence de la fréquence de fauche sur la production de biomasse et la composition chimique du foin de Pennisetum clandestinum
}

\author{
Z. KAMBALE MUHINDO, F. TENDONKENG ${ }^{*}$, E. MIEGOUE et \\ E. PAMO TEDONKENG
}
Laboratoire de Production et de Nutrition Animales, Département des Productions Animales, Faculté d'Agronomie et des Sciences Agricoles (FASA), Université de Dschang.
B P 222 Dschang, Cameroun.
*Auteur correspondant ; E-mail: f.tendonkeng@univ-dschang.org

\section{RESUME}

L'influence de la fréquence de fauche sur la production de biomasse et la composition chimique du foin de Pennisetum clandestinum a été étudiée entre mars 2015 et mars 2016 à l'Université de Dschang. L'objet de l'étude était de contribuer à l'amélioration de la gestion d'une culture de $P$. clandestinum en vue d'assurer un disponible fourrager permanent en quantité et qualité optimales par le recours à la fenaison. A cet effet, un dispositif expérimental en bloc complètement randomisé comparant trois fréquences de fauche (30, 45 et 60 jours de croissance) en 8 répétitions sur des planches de $4 \mathrm{~m}^{2}(2 \mathrm{~m} \times 2 \mathrm{~m})$ chacune, soit un total de 24 planches par bloc, a été utilisé pour la culture de $P$. clandestinum. Le fourrage a été successivement fauché à chaque fréquence durant une saison culturale. Des échantillons de fourrage ont été prélevés à chaque fauche pour l'évaluation de la biomasse tandis que le reste du fourrage a été séché au soleil pour la production du foin. Des échantillons de foin de chaque fréquence de fauche ont été analysés pour la détermination de la composition chimique. Les résultats obtenus ont montré que l'augmentation de l'intervalle de fauche (30, 45 et 60 jours) a entraîné une augmentation non significative $(\mathrm{p}>0,05)$ de la biomasse totale $(16,10 ; 18,64$ et 19,41 t MS/ha) et significative $(\mathrm{p}<0,05)$ de la biomasse moyenne $(2,68 ; 4,66$ et $6,47 \mathrm{t} \mathrm{MS} / \mathrm{ha})$. La composition chimique du fourrage a montré que l'augmentation de l'intervalle de fauche a eu pour effet l'augmentation significative $(\mathrm{p}<0,05)$ des teneurs en MS, MO, NDF, cellulose, hémicellulose et glucides totaux. La tendance inverse a été observée avec les cendres, les protéines brutes, la digestibilité de la matière organique, les matières azotées digestibles, les unités fourragères lait et viande. Enfin, la teneur en lipides la plus élevée $(2,89 \%)$ a été obtenue avec la fréquence de 45 jours. Tenant compte, à la fois, de la quantité (biomasse produite) et de la qualité (composition chimique), il ressort de cette étude que la fauche de 45 jours constitue l'option de choix d'exploitation optimale de la culture de $P$. clandestinum pour une fenaison.

(C) 2018 International Formulae Group. All rights reserved.

Mots clés: Biomasse, composition chimique, foin, intervalle de fauche, Pennisetum clandestinum.

\section{Influence of cutting frequency on biomass production and chemical composition of Pennisetum clandestinum hay}

\begin{abstract}
The cutting frequency effect on biomass production and chemical composition of Pennisetum clandestinum hay was studied between March 2015 and March 2016 at the University of Dschang. This study main objective was to contribute to the improvement of $P$. clandestinum crop availability in optimal quantity
\end{abstract}


and quality permanently through haymaking. For this purpose, a completely randomized block design comparing three cutting frequencies (30, 45 and 60 days of growth) in 8 replicates on plots of $4 \mathrm{~m}^{2}(2 \mathrm{~m} \mathrm{x} 2 \mathrm{~m})$ each one, for a total of 24 plots per block, was used for $P$. clandestinum cultivation. Consecutive cuttings at each frequency were done throughout the annual farming season. Samples of forage at each harvest were collected for biomass evaluation while the main crop was sun-dried for hay making. Samples of hay from each cutting frequency were analyzed for the chemical composition evaluation. Results showed that the effect of increasing the cutting interval (30, 45 and 60 days) on biomass production was the increase $(\mathrm{p}<0.05)$ of yield average $(2.68 ; 4.66$ and $6.47 \mathrm{t} \mathrm{MS/ha),} \mathrm{but} \mathrm{no} \mathrm{significant}(\mathrm{p}>0.05)$ effect was observed on the total yield increment (16.10; 18.64 and $19.41 \mathrm{t} \mathrm{MS/ha).} \mathrm{Chemical} \mathrm{composition} \mathrm{of} \mathrm{the} \mathrm{forage} \mathrm{showed} \mathrm{that} \mathrm{DM,} \mathrm{OM,} \mathrm{NDF,}$ cellulose, hemicellulose and total carbohydrate contents increased; while ash and crude proteins contents, organic matter digestibility, digestible crude proteins, energy for milk and for meat production decreased. In addition, the highest lipid content $(2.89 \%)$ was obtained at the 45 days frequency. Finally, considering both the quantity (biomass produced) and the quality (chemical composition) of forage throughout this study, the 45 days cutting frequency should constitute the better management option of $P$. clandestinum crop for haymaking. (C) 2018 International Formulae Group. All rights reserved.

Keywords: Biomass, chemical composition, cutting interval, hay, Pennisetum clandestinum.

\section{INTRODUCTION}

L'alimentation est l'une des principales causes de la faible productivité des ruminants en région tropicale. Dans la plupart des élevages traditionnels, particulièrement des herbivores domestiques en Afrique au sud du Sahara, l'animal est nourri sur des parcours naturels (Pamo et al., 2006 ; Babatounde et al., 2011). Les zones de pâturages naturels disponibles pour les petits paysans diminuent rapidement à mesure que les terres cultivées avancent (Pamo et al., 2007 ; Kiema et al., 2012). La faible disponibilité d'aliments en quantité et en qualité, particulièrement en saison sèche, affecte négativement l'état de santé des animaux et leurs productions (Suttie, 2004 ; Tshibangu et al., 2014). Par ailleurs, les prospectives offre-demande en produits animaux en Afrique subsaharienne pour 2020 évaluent à 17,39 millions de tonnes (équivalent carcasse) la demande totale en viande (pour une population estimée à 1041 millions d'habitants) alors que la production sera de 14,405 millions de tonnes, soit un déficit de 2,985 millions de tonnes. L'offre de viande rouge (bovins et petits ruminants) qui satisfait $55,6 \%$ de la consommation actuelle totale de viande n'en satisferait plus que $30,9 \%$ en 2020 (FAO, 2000). Eu égard à l'accroissement démographique et à l'élévation du niveau de vie, la production de viande constitue une priorité pour satisfaire une demande sans cesse croissante en produits carnés au sud du Sahara (Babatounde et al., 2011).

C'est par les cultures fourragères que les contraintes alimentaires pourront être partiellement levées. Elles sont de plus en plus nécessaires face à l'évolution du milieu et à la diminution des ressources pastorales naturelles. Les cultures fourragères sont d'une importance capitale dans le développement durable de l'élevage (César et al., 2004). Les fluctuations saisonnières dans la croissance et la maturité de la plante rendent indispensables la récolte et le stockage du fourrage afin de maximiser la qualité et la productivité (Decruyenaere et al., 2005). En Afrique tropicale, la constitution de réserves fourragères par fenaison semble être une voie appropriée pour l'amélioration de la productivité animale (César et al., 2004). Selon Chapoutot et al. (2009), la connaissance de la valeur alimentaire (dont la composition chimique) d'un aliment destiné aux animaux d'élevage est indispensable pour garantir une valorisation optimale de ce produit. Etant donné que chaque type de plante a une valeur alimentaire propre (teneur en énergie, protéine, minéraux, digestibilité, ...) qui évolue au cours du temps, la récolte d'un fourrage réalisée au stade optimum et le choix judicieux d'une ou plusieurs techniques de conservation sont des éléments fondamentaux 
de réussite en production fourragère (Crémer et Knoden, 2012). Au regard d'une telle évolution, Pennisetum clandestium dans la présente étude, a été fauché à différents âges de croissance et conservé à l'état de foin. L'objectif de cette étude est de déterminer la maturité à laquelle $P$. clandestinum pourrait être fauché fréquemment et permettant d'obtenir du foin en quantité et composition chimique optimales.

\section{MATERIEL ET METHODES \\ Zone de l'étude}

L'étude s'est déroulée entre Mars 2015 et Mars 2016 à la Ferme d'Application et de Recherche (FAR) et au Laboratoire de Production et de Nutrition Animales (LAPRONAN) de l'Université de Dschang. Située à une altitude d'environ $1420 \mathrm{~m}$ et localisée entre $10^{\circ} 03^{\prime}$ de longitude Est et $05^{\circ} 26^{\prime}$ de latitude Nord, cette zone reçoit entre 1500 et $2000 \mathrm{~mm}$ de pluie par an. Les températures varient en moyenne entre $16{ }^{\circ} \mathrm{C}$ à $21{ }^{\circ} \mathrm{C}$, avec un maximum de $31{ }^{\circ} \mathrm{C}$ durant le mois le plus chaud. Le climat est de type soudano-guinéen d'altitude, avec une courte saison sèche (mi-novembre à mi-mars) et une longue saison de pluies (mi-mars à minovembre) correspondant à la période des cultures (Pamo et al., 2005).

\section{Dispositif expérimental et analyse du sol}

Un dispositif en bloc complètement randomisé comparant trois fréquences de fauche (30, 45 et 60 jours de croissance) en huit répétitions sur des planches de $4 \mathrm{~m}^{2}(2 \mathrm{~m}$ x $2 \mathrm{~m}$ ) chacune, soit un total de 24 planches par bloc, a été utilisé. Les échantillons du sol ont été prélevés sur le site expérimental dans l'horizon $0-20 \mathrm{~cm}$ avant la préparation du terrain et la mise en place des boutures de $P$. clandestinum. L'analyse a été effectuée au Laboratoire d'Analyse des Sols, de Chimie et de l'Environnement (LABASCE) de l'Université de Dschang. Les résultats ont montré que ce sol était limoneux, légèrement acide, renfermant $12 \%$ de matière organique, $7 \%$ de carbone, $0,6 \%$ d'azote total et 0,5 $\mathrm{mg} / \mathrm{kg}$ de phosphore assimilable.

\section{Préparation du terrain, plantation et entretien de la culture}

La préparation du terrain a consisté principalement au défrichage manuel et au labour mécanisé. En vue d'améliorer la productivité du sol, un engrais de fond constitué de crottes sèches de chèvres (à $16,80 \%$ N) a, au regard des résultats de l'analyse du sol et en fonction de besoins azotés de la culture, été incorporé au sol à raison de $1,19 \mathrm{~kg} / \mathrm{m}^{2}$, soit $19,992 \mathrm{~g}$ d'azote $/ \mathrm{m}^{2}$ (environ $200 \mathrm{~kg} \mathrm{~N} / \mathrm{ha}$ ). La plantation des boutures a été faite manuellement. Les boutures d'environ $30 \mathrm{~cm}$ de long comportant au moins 4 bourgeons végétatifs par bouture, ont été plantées aux écartements de $20 \mathrm{~cm} \mathrm{x}$ $20 \mathrm{~cm}$, par l'enfouissement de 2 à 3 bourgeons végétatifs dans le sol; soit une densité de 121 boutures par planche. L'entretien de la culture a consisté au contrôle des adventices par sarclage manuel fait mensuellement durant toute la période de l'essai.

\section{Fauches successives et fenaison}

$\mathrm{Au}$ dernier jour de chaque fréquence de fauche $\left(30^{\text {ème }}, 45^{\text {ème }}\right.$ et $60^{\text {ème }}$ jour de croissance), le fourrage a été récolté sur 8 planches par bloc. La coupe a été faite à la hauteur de $10 \mathrm{~cm}$ du sol. Les repousses de $P$. clandestinum ont permis de réaliser, au cours de la saison culturale, respectivement 6,4 et 3 récoltes aux fréquences de 30,45 et 60 jours. Le fourrage récolté était étalé sur des nattes en vue du séchage au soleil. Pour le garder à l'abri des intempéries, deux aires de séchage de $17,0 \mathrm{~m}^{2}(7,4 \mathrm{~m} \mathrm{x} 2,3 \mathrm{~m})$ et $23,5 \mathrm{~m}^{2}(8,7 \mathrm{~m} \mathrm{x}$ $2,7 \mathrm{~m}$ ) ont été érigées sur pilotis (environ 0,5 $\mathrm{m}$ du sol) et couvertes de bâches amovibles en polyéthylène. En fonction de l'intensité et de la durée du rayonnement solaire quotidien, le fourrage sec (qui est le foin) était obtenu au bout de 7 à 10 jours pour les fauches faites en pleine saison de pluie, et 3 jours pour les dernières fauches intervenues en fin de saison de pluie.

\section{Evaluation de la biomasse}

Des échantillons de fourrage ont été prélevés à chaque récolte pour l'évaluation de la biomasse. A cet effet, 4 planches ont été 
choisies au hasard et la coupe y a été faite à la hauteur de $10 \mathrm{~cm}$ du sol sur une aire utile de 1 $\mathrm{m}^{2}$ délimitée au centre de chaque planche par une table de fauche métallique. Le fourrage récolté était immédiatement pesé à l'aide d'une balance électronique de marque Dahongying, de portée $30 \mathrm{~kg}$ et de précision 2 $\mathrm{g}$, pour la détermination de la biomasse fraîche. Un échantillon de $500 \mathrm{~g}$ de ce fourrage était prélevé sur chacune des 4 planches et séché à $60{ }^{\circ} \mathrm{C}$ dans une étuve ventilée jusqu'à un poids constant à partir duquel la biomasse sèche a été calculée ainsi que le rendement (t MS/ha).

\section{Evaluation de la composition chimique}

Les foins de toutes les fauches d'une fréquence ont d'abord été convenablement mélangés. Les échantillons prélevés dans chaque mélange étaient moulus à l'aide d'un broyeur tri-marteaux muni d'un tamis aux mailles de $1 \mathrm{~mm}$ de diamètre. Les poudres obtenues étaient gardées dans des sachets en polyéthylène en vue de différentes analyses bromatologiques. La détermination des teneurs en matière sèche (MS), en cendres, en matière organique $(\mathrm{MO})$, en lipides et en protéines brutes $(\mathrm{PB})$ a été effectuée selon les méthodes décrites par AOAC (2005). Quant aux parois cellulaires (NDF, ADF et ADL), leur détermination s'est faite selon la méthode décrite par Van Soest et al. (1991).

\section{Analyses statistiques}

Les données sur la biomasse produite et la composition chimique des foins ont été soumises à une analyse de variance à un facteur, suivant le modèle linéaire général, à l'aide du logiciel SPSS version 23. Lorsque les différences existaient entre les traitements, les moyennes ont été séparées par le test de Duncan au seuil de $5 \%$.

\section{RESULTATS}

\section{Effet de la fréquence de fauche sur la biomasse}

La variation de la biomasse sèche produite aux fauches successives de chaque fréquence de fauche montre que la biomasse produite à la fréquence de 30 jours a connu d'abord une diminution de la première à la quatrième fauche, ensuite une augmentation aux deux dernières fauches. De la $2^{\text {ère }}$ à la $6^{\text {ème }}$ fauche, les rendements en biomasse ont été comparables $(p>0,05)$ mais significativement $(\mathrm{p}<0,05)$ inférieurs à celui de la $1^{\text {ère }}$ fauche. La même tendance a été observée avec la fréquence de 45 jours. Par contre, à la fréquence de 60 jours, la biomasse produite a seulement diminué de la première à la dernière fauche; les rendements des deux dernières fauches ont été comparables $(p>0,05)$ et significativement $(p<0,05)$ inférieurs à celui de la $1^{\text {ère }}$ fauche (Figure 1).

La biomasse moyenne correspondant à chaque fréquence de fauche a significativement $(\mathrm{p}<0,05)$ augmenté avec l'augmentation de l'intervalle de fauche. Par contre, aucun effet significatif n'a été observé sur la biomasse totale (biomasse cumulée des fauches successives de chaque fréquence de fauche) (Tableau 1).

\section{Effet de la fréquence de fauche sur la composition chimique et la teneur en nutriments des foins}

La composition chimique des foins de $P$. clandestinum a montré que l'augmentation de l'intervalle de fauche a eu pour effet l'augmentation des teneurs en MS, MO, NDF, cellulose et hémicellulose; les teneurs obtenues à la fréquence de 60 jours sont significativement $(\mathrm{p}<0,05)$ plus élevées que celles obtenues à la fréquence de 30 jours. La tendance inverse a été observée avec les cendres et les protéines brutes (PB) tandis que la teneur en lipides la plus élevée a été obtenue avec la fréquence de 45 jours, comparable $(p>0,05)$ à la fréquence de 30 jours (Tableau 2).

Les teneurs en nutriments calculées à partir de la composition chimique des foins ont montré que les glucides totaux ont significativement $(\mathrm{p}<0,05)$ augmenté tandis que la digestibilité de la matière organique (dMO), les matières azotées digestibles (MAD), les unités fourragères lait (UFL) et viande (UFV) ont diminué avec l'augmentation de l'intervalle de fauche (Tableau 3). 
F30

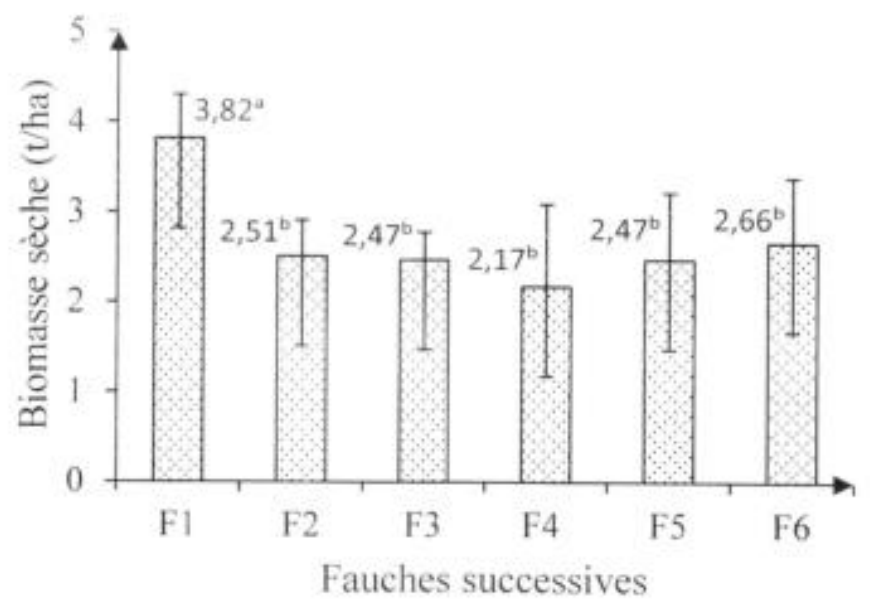

F45

F60
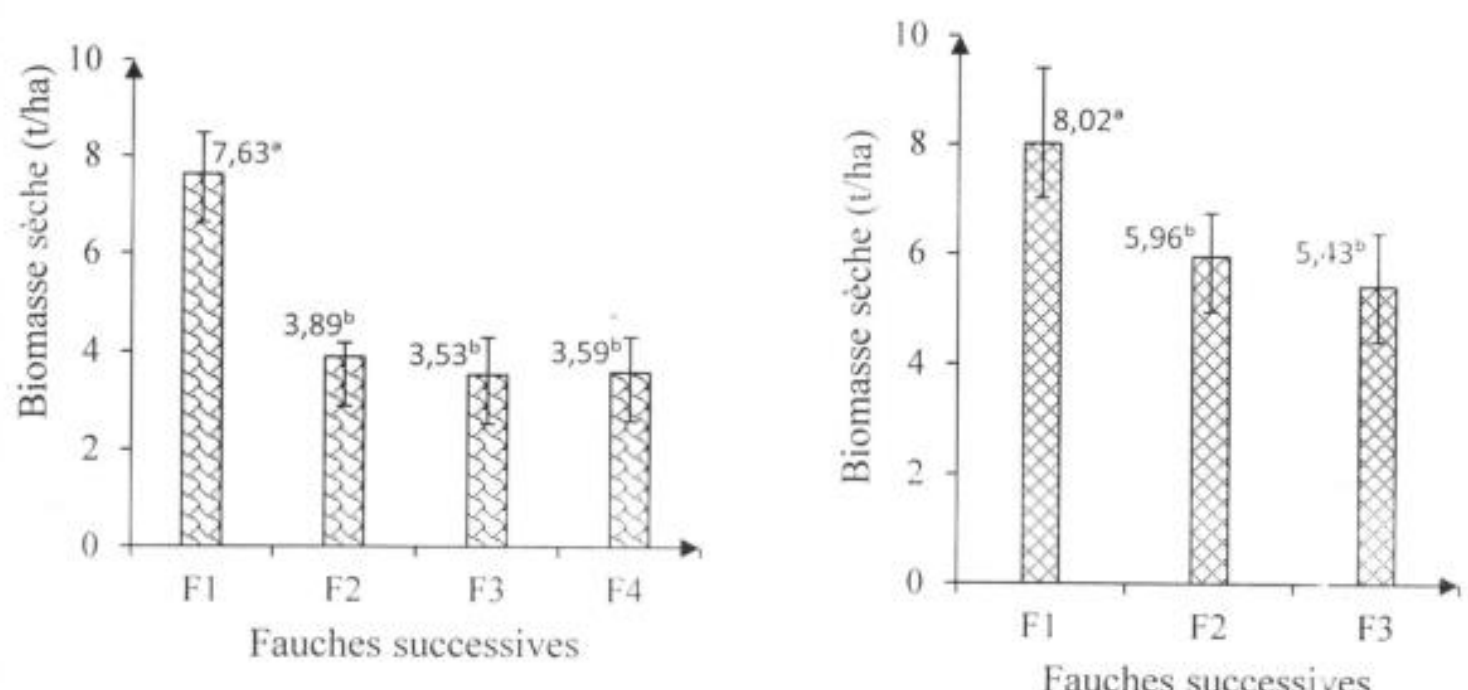

Figure 1 : Evolution de la production de la biomasse sèche des fauches successives de P. clandestinum aux fréquences de 30 jours (F30), 45 jours (F45) et 60 jours (F60). $\mathrm{F} 1=$ Première fauche $; \mathrm{F} 2=$ Deuxième fauche $; \mathrm{F} 3=$ Troisième fauche $; \mathrm{F} 4=$ Quatrième fauche ; $\mathrm{F} 5=$ Cinquième fauche $; \mathrm{F} 6=$ Sixième fauche. t/ha: tonne par hectare.

a, b: les moyennes portant la même lettre sur les histogrammes d'une même fréquence de fauche sont statistiquement comparables au seuil de 5\%. 
Tableau 1: Biomasses moyenne et totale par fréquence de fauche

\begin{tabular}{cccc}
\hline $\begin{array}{c}\text { Fréquence de } \\
\text { fauche (jours) }\end{array}$ & $\begin{array}{c}\text { Nombre } \\
\text { de fauches }\end{array}$ & $\begin{array}{c}\text { Biomasse totale } \\
(\mathbf{t} / \mathbf{h a})\end{array}$ & $\begin{array}{c}\text { Biomasse moyenne } \\
(\mathbf{t} / \mathbf{h a})\end{array}$ \\
\hline $\mathbf{3 0}$ & 6 & $16,10 \pm 1,73^{\mathrm{a}}$ & $2,68 \pm 0,29^{\mathrm{a}}$ \\
$\mathbf{4 5}$ & 4 & $18,64 \pm 3,36^{\mathrm{a}}$ & $4,66 \pm 0,84^{\mathrm{b}}$ \\
$\mathbf{6 0}$ & 3 & $19,41 \pm 2,49^{\mathrm{a}}$ & $6,47 \pm 0,83^{\mathrm{c}}$ \\
\hline
\end{tabular}

$\mathrm{t} / \mathrm{ha}=$ tonne par hectare.

a, b, c: les moyennes portant la même lettre dans la même colonne sont statistiquement comparables au seuil de 5\%.

Tableau 2: Composition chimique des foins de P. clandestinum à différentes fréquences de fauche

\begin{tabular}{lcccccc}
\hline \multirow{2}{*}{$\begin{array}{c}\text { Composition chimique } \\
(\% \text { MS })\end{array}$} & \multicolumn{2}{c}{ Fréquence de fauche (jours) } & & ESM & $\boldsymbol{p}$ \\
\cline { 2 - 4 } & $\mathbf{3 0}$ & $\mathbf{4 5}$ & $\mathbf{6 0}$ & & \\
\hline Matière sèche $\left(^{*}\right)$ & $96,03^{\mathrm{a}}$ & $96,86^{\mathrm{ab}}$ & $97,09^{\mathrm{b}}$ & 0,43 & 0,035 \\
Cendres & $12,01^{\mathrm{a}}$ & $11,10^{\mathrm{b}}$ & $10,65^{\mathrm{b}}$ & 0,17 & 0,001 \\
Matière organique & $87,99^{\mathrm{a}}$ & $88,90^{\mathrm{b}}$ & $89,35^{\mathrm{b}}$ & 0,17 & 0,001 \\
Protéines brutes & $21,46^{\mathrm{a}}$ & $15,66^{\mathrm{b}}$ & $14,59^{\mathrm{b}}$ & 0,52 & 0,000 \\
Lipides & $2,88^{\mathrm{a}}$ & $2,89^{\mathrm{a}}$ & $1,72^{\mathrm{b}}$ & 0,20 & 0,003 \\
NDF & $77,41^{\mathrm{a}}$ & $88,95^{\mathrm{b}}$ & $91,25^{\mathrm{b}}$ & 2,27 & 0,004 \\
Cellulose & $26,43^{\mathrm{a}}$ & $28,35^{\mathrm{ab}}$ & $31,67^{\mathrm{b}}$ & & 1,24 & 0,043 \\
Hémicellulose & $41,82^{\mathrm{a}}$ & $52,45^{\mathrm{b}}$ & $53,93^{\mathrm{b}}$ & & 2,40 & 0,012 \\
\hline
\end{tabular}

(*): \% échantillon.

$\mathrm{NDF}=$ Neutral detergent fibre ; ESM : Erreur standard sur la moyenne ; $\mathrm{p}$ : Probabilité.

a, b: les moyennes portant la même lettre sur la même ligne sont statistiquement comparables au seuil de 5\%.

Tableau 3 : Teneur en nutriments des foins de $P$. clandestinum à différentes fréquences de fauche

\begin{tabular}{lccccc}
\hline \multirow{2}{*}{ Teneur en nutriments } & \multicolumn{3}{c}{ Fréquence de fauche (jours) } & & \multirow{2}{*}{$\mathbf{E S M}$} \\
\cline { 2 - 4 } & $\mathbf{3 0}$ & $\mathbf{4 5}$ & $\mathbf{6 0}$ & & $\boldsymbol{P}$ \\
\hline Glucides totaux (\% MS) & $63,66^{\mathrm{a}}$ & $70,36^{\mathrm{b}}$ & $73,05^{\mathrm{c}}$ & 0,57 & 0,000 \\
dMO (\% MS) & $41,31^{\mathrm{a}}$ & $37,27^{\mathrm{ab}}$ & $30,29^{\mathrm{b}}$ & 2,60 & 0,042 \\
MAD (g/100g MO) & $16,14^{\mathrm{a}}$ & $11,28^{\mathrm{b}}$ & $10,39^{\mathrm{b}}$ & 0,43 & 0,000 \\
UFL (kg/MS) & $0,80^{\mathrm{a}}$ & $0,76^{\mathrm{a}}$ & $0,68^{\mathrm{b}}$ & 0,02 & 0,017 \\
UFV (kg/MS) & $0,71^{\mathrm{a}}$ & $0,67^{\mathrm{a}}$ & $0,57^{\mathrm{b}}$ & 0,03 & 0,019 \\
\hline
\end{tabular}

$\mathrm{dMO}=$ digestibilité de la matière organique $; \mathrm{MAD}=$ matière azotée digestible $; \mathrm{UFL}=$ unité fourragère pour le lait ;

UFV = unité fourragère pour la viande.

a, b, c: les moyennes portant la même lettre sur la même ligne sont statistiquement comparables au seuil de 5\%. 


\section{DISCUSSION}

\section{Effet de la fréquence de fauche sur la} production de biomasse de $P$. clandestinum

La fréquence de fauche a eu pour effet, au niveau des fauches successives, la diminution du rendement de biomasse indépendamment de la fréquence de fauche. Toutefois, aux fréquences de 30 et 45 jours, la diminution du rendement de la $1^{\text {ère }}$ à la $4^{\text {ème }}$ fauche et de la $1^{\text {ère }}$ à la $3^{\text {ème }}$ fauche respectivement, a été suivie par une augmentation aux fauches suivantes. Cette inflexion pourrait être un effet de variation saisonnière étant donné que les fauches affectées par l'augmentation ont eu lieu en fin de saison de pluie lorsque la pluviosité avait sensiblement diminué. Une observation similaire a été faite par Guérin (1999) qui a rapporté que le rendement en matière sèche à l'hectare et par jour des parties aériennes de Panicum maximum en culture intensive au Sénégal était plus élevé en saison chaude qu'en saison pluvieuse. A la fréquence de 60 jours, le rendement en biomasse sèche a diminué de la première à la dernière fauche. Globalement, la diminution du rendement observée aux fauches postérieures à la première pourrait être due à la réduction progressive de la fertilité du sol suite aux exportations des éléments minéraux par les récoltes consécutives. En effet, en dehors de l'engrais de fond constitué de crottes de chèvre, la culture de $P$. clandestinum n'a bénéficié d'aucune autre forme de fertilisation compensatrice des exportations minérales par les parties aériennes récoltées. Zoffoun et al. (2013) ont également rapporté que, dans les pâturages artificiels en zone soudanienne et subéquatoriale, l'élévation de l'intensité de pâture s'accompagnait de la diminution de la production de biomasse de Panicum maximum var. $\mathrm{C} 1$. Ce résultat et celui de la présente étude corroborent l'assertion de nombreux auteurs (Cremer et Knoden, 2012 ; Cremer, 2014) selon laquelle la vitesse et l'importance de la repousse d'une graminée pérenne pâturée ou fauchée fréquemment vont dépendre du niveau des réserves mobilisables accumulées ou détenues dans les parties ayant échappé au prélèvement, de l'activité photosynthétique des parties vertes restantes et du sol (eau, éléments fertilisants).
S'agissant de la production cumulée de biomasse par fréquence de fauche, l'augmentation de l'intervalle de fauche (30, 45 et 60 jours) a entraîné une augmentation non significative de la biomasse totale $(16,10$; 18,64 et 19,41 t MS/ha) et significative de la biomasse moyenne $(2,68 ; 4,66$ et $6,47 \mathrm{t}$ $\mathrm{MS} / \mathrm{ha}$ ). L'augmentation du rendement de biomasse en fonction de l'augmentation de l'intervalle de fauche se justifierait principalement par la maturité à la récolte. A cet effet, Tendonkeng et al. (2009) ont souligné que l'augmentation du rendement en biomasse sèche en fonction de la maturité de la plante est une résultante de l'élongation des feuilles, du développement des tiges ou de nouvelles feuilles. Par conséquent, de plus longs intervalles de fauche (45 et 60 jours) sont caractérisés par une augmentation du taux moyen de croissance de la plante durant la phase végétative.

\section{Effet de la fréquence de fauche sur la composition chimique des foins de $P$. clandestinum}

L'effet de la fréquence de fauche sur la composition chimique des foins de $P$. clandestinum a varié d'un constituant à l'autre. Le résultat de chaque fréquence de fauche indique que le taux de MS, MO, NDF, cellulose et hémicellulose a augmenté de la plus courte à la plus longue fréquence de fauche. A un âge croissant de récolte de $P$. clandestinum, soit 50, 70 et 90 jours, Ruggia et al. (2008) ont obtenu une augmentation du taux de MO respectivement de 88,9 ; 90,8 et $91,5 \%$ dans les foins. Ce résultat ainsi que celui de la présente étude se justifieraient par le développement des tissus et organes de la plante en fonction de l'âge, lequel s'est traduit aussi bien par une augmentation du taux de MS que de MO. Les teneurs en parois cellulaires (NDF), en cellulose et en hémicellulose ont augmenté de la plus courte à la plus longue fréquence de fauche. Ces résultats sont en accord avec ceux de Ruggia et al. (2008) qui ont rapporté, pour les foins de $P$. clandestinum, une augmentation de $65,4 \%$ à $67,5 \%$ NDF et 25,2 à $30,6 \%$ cellulose aux stades de récolte de 50 et 90 jours respectivement. Quant à l'hémicellulose, ces auteurs ont rapporté une diminution de $34,0 \%$ 
à 31,8\%. Conformément à plusieurs auteurs (Fukumoto et Lee, 2003; Holliday, 2007 ; Crémer et Knoden, 2012; Ramirez et al., 2015), les résultats de la présente étude s'expliqueraient par le fait que le contenu pariétal (NDF, cellulose, hémicellulose, lignine) augmente avec la maturité de la plante pour la plupart des fourrages. Etant une plante $\mathrm{C} 4, P$. clandestinum est caractérisé, comme toutes les graminées tropicales $\mathrm{C} 4$, par une teneur élevée en fibres, la croissance étant stimulée et la sénescence accélérée par les températures et les précipitations élevées du climat tropical.

Par ailleurs, l'augmentation de l'intervalle de fauche s'est traduite par une diminution du taux de cendres et de protéines brutes. La diminution du taux de cendres converge avec le résultat rapporté par Ruggia et al. (2008) à savoir une diminution de 11,3 à $11,0 \%$ de cendres dans les foins de $P$. clandestinum récolté respectivement à 50 et 90 jours de croissance. Dans la présente étude, la diminution progressive de la teneur en cendres en fonction de la maturité de la plante pourrait se justifier par un faible taux d'absorption minérale suite à l'épuisement continu des réserves minérales du sol. Concernant les protéines brutes, Ramirez et al. (2015) ont obtenu une diminution de la teneur en protéines de 25,4 à $17,0 \%$ avec l'augmentation de l'âge de fauche de $P$. clandestinum de 30 à 60 jours. L'évolution décroissante de la teneur en PB des fourrages se justifierait par le fait qu'avec l'augmentation de l'âge de la plante, les tiges prennent de plus en plus d'ampleur par rapport aux feuilles, les teneurs en parois cellulaires augmentent et les teneurs en PB diminuent au profit de la cellulose brute (Pamo et al., 2005).

Les teneurs en lipides aux fréquences de 30 et 45 jours ont été comparables et significativement plus élevées que celle de la fréquence de 60 jours. Les teneurs plus élevées sont proches de la moyenne de 2,7\% rapportée par Heuzé et al. (2015). Pour nombreux auteurs (Morel et al., 2006 ; Arrigo, 2010), la teneur en lipides du fourrage diminue avec le stade de développement des plantes et le procédé de conservation suite, notamment, à des phénomènes de pertes de feuilles et par respiration. Afin de sauvegarder les teneurs en lipides, ces auteurs suggèrent que les plantes soient récoltées jeunes et le fanage réalisé rapidement tout en ménageant le fourrage pour conserver les nutriments contenus dans les feuilles.

Concernant la teneur en nutriments, la fréquence de fauche a influencé positivement les glucides totaux qui ont augmenté significativement avec l'augmentation de l'intervalle de fauche; tandis que la $\mathrm{dMO}$, la MAD, l'UFL et l'UFV ont significativement diminué de la plus courte à la plus longue fréquence de fauche. Pour la $\mathrm{dMO}$, ce résultat est en accord avec celui de Ruggia et al. (2008) qui ont obtenu une baisse de la dMO des foins de $P$. clandestinum, soit 53,1;50,4 et $47,4 \%$ respectivement à 50,70 et 90 jours de fauche. Etant donné que la MAT a baissé $\mathrm{du}$ plus petit au plus grand intervalle de fauche dans la présente étude, la MAD a varié de la même manière. Quant aux unités fourragères pour le lait et la viande (UFL et UFV), selon Baumont et al. (2009), la valeur énergétique des fourrages s'exprimant par leur teneur en énergie nette dans le système des unités fourragères (UFL, UFV), le principal facteur de leur variation est la digestibilité de l'énergie brute des aliments qui est très étroitement liée à la digestibilité de la matière organique (dMO). Cette dernière ayant diminué, dans la présente étude, de la fréquence de fauche de 30 jours à celle de 60 jours, les valeurs UFL et UFV ont évolué de la même manière.

\section{Conclusion}

$\mathrm{Au}$ terme de cette étude portant sur l'influence de la fréquence de fauche sur la production de biomasse et la composition chimique de $P$. clandestinum, l'augmentation de l'intervalle de fauche a eu pour effet :

- sur la biomasse: une augmentation significative de la biomasse moyenne et non significative de la biomasse totale produite ;

- sur la composition chimique et la teneur en nutriments des foins: une augmentation significative du taux de MS, de MO, de NDF, de cellulose, d'hémicellulose et des glucides totaux; une diminution significative de la teneur en cendres, en PB 
ainsi que la dMO, la MAD et les unités fourragères lait (UFL) et viande (UFV) notamment entre la plus courte et la plus longue fréquence de fauche.

En associant les exigences d'une production quantitative et qualitative optimales d'une culture fourragère, la fauche de 45 jours s'avère une option de choix d'exploitation, dans les conditions de cette étude, de la culture de $P$. clandestinum pour une fenaison.

\section{CONFLIT D'INTERETS}

Les auteurs déclarent, sur l'honneur, qu'il n'existe aucun conflit d'intérêt ni entre eux, ni avec une structure ou organisation quelconque par rapport au présent article.

\section{CONTRIBUTIONS DES AUTEURS}

ZKM et FT ont contribué à la mise en place du dispositif expérimental, la collecte et traitement des données, la rédaction de l'article. EM a contribué à la mise en place du dispositif expérimental et la collecte des données. EPT a supervisé toutes les activités de collecte de données et la rédaction de l'article.

\section{REMERCIEMENTS}

Les auteurs remercient l'Université de Dschang pour la mise à leur disposition du cadre approprié de recherche (Ferme d'Application et de Recherche ainsi que le Laboratoire de Production et de Nutrition Animales).

\section{REFERENCES}

AOAC (Association of Official Analytical Chemists). 2005. Official Methods of Analysis (18 ${ }^{\text {th }}$ edn). AOAC International: Washington, D.C.; 180 p.

Arrigo Y. 2010. Matière grasse et composition en acides gras des fourrages conservés. Rech. Agron. Suisse, 1(10): 366-371.

Babatounde S, Sidi H, Houinato M, Oumorou M, Mensah GA, Sinsin BA. 2011. Valeur alimentaire des fourrages consommés par les taurillons Borgou sur les parcours naturels du centre du Bénin. Int. J. Biol. Chem. Sci., 5(6): 2382-2394. DOI : http://dx.doi.org/10.4314/ijbcs.v5i6.18
Baumont R, Aufrère J, Meschy F. 2009. La valeur alimentaire des fourrages : rôle des pratiques de culture, de récolte et de conservation. Fourrages, 198: 153-173.

César J, Ehouinsou M, Gouro A. 2004. Production fourragère en zone tropicale et conseils aux éleveurs. PROCORDEL (Conseils et formation en appui à la production laitière). $48 \mathrm{p}$.

Chapoutot P, Leclerc MC, Brunschwig P, Boulan P. 2009. Guide pour la prévision de la valeur nutritive des coproduits pour les ruminants. Institut d'Elevage/Chambre d'Agriculture ; Paris. $50 \mathrm{p}$.

Crémer S. 2014. Introduction à la reconnaissance des graminées. Fourrages Mieux ASB ; Marloie ; Belgique. 6 p.

Crémer S, Knoden D. 2012. Influence du stade de développement des plantes sur la qualité des fourrages récoltés. Fourrages Mieux ASBL; Marloie; Belgique. $2 \mathrm{p}$.

Decruyenaere V, Stilmant D, Agneessens R, Buldgen A, Dardenne P, Bartiaux-Thill N. 2005. Les productions fourragères : vers une gestion et une utilisation optimales des ressources locales. Publications «Fourrages Mieux»; Centre d'Economie Rural à Marloie/Wallonie ; Belgique. 7 p.

FAO. 2000. Annuaire de la production 1999. Rome; Italie. Vol. 53 ; 246 p.

Fukumoto GK, Lee CN. 2003. Kikuyu grass for forage. Livestock Management 5, Cooperative Extension Service, College of tropical agriculture and human resources (CTAHR), Manoa/Hawaï. 4 p.

Guérin H. 1999. Valeur alimentaire des fourrages cultivés. In Cultures Fourragères Tropicales, Roberge G, Toutain B (eds). CIRAD ; 93-145.

Heuzé V, Tran G, Boval M. 2015. Kikuyu (Pennisetum clandestinum). Feedipedia, a programme by INRA, CIRAD, AFZ and FAO. Datasheet online; $13 \mathrm{p}$. http://feedipedia.org/node/398

Holliday J. 2007. Management of Kikuyu (Pennisetum clandestinum) for Improved Dairy Production. Grassland Science: Pietermaritzburg, South Africa; 189 p. 
Kiema A, Sawadogo I, Ouédraogo T, Aimé Joseph Nianogo AJ. 2012. Stratégies d'exploitation $\mathrm{du}$ fourrage par les éleveurs de la zone sahélienne du Burkina Faso. Int. J. Biol. Chem. Sci., 6(4): $\quad 1492-1505 . \quad$ DOI: http://dx.doi.org/10.4314/ijbcs.v6i4.8

Morel I, Wyss U, Collomb M, Bütikofer U. 2006. Influence de la composition botanique de l'herbe ou du foin sur la composition du lait. Rev. suisse Agric., 38(1): 9-15.

Pamo TE, Boukila B, Fonteh FA, Tendonkeng F, Kana JR, Nanda AS. 2007. Nutritive values of some basic grasses and leguminous tree foliage of the Central region of Africa. Anim. Feed Sci. Technol., 135: 273-282. http://doi.org/10.1016/j.anifeedsci.2006. 07.001

Pamo TE, Fonteh FA, Tendonkeng F, Kana JR, Djaga PJ, Fomewang II G. 2006. Influence of supplementary feeding of multipurpose leguminous tree leaves on kid growth and milk production of the West African Dwarf goats. Small Rum. Res., $\quad$ 63: 142-149. http://doi.org/10.1016/j.smallrumres.200 5.05.011

Pamo TE, Boukila B, Fonteh FA, Tendonkeng F, Kana JR. 2005. Composition chimique et effets de la supplémentation avec Calliandra calothyrsus et Leucaena leucocephala sur la production laitière et la croissance des chevreaux nains de Guinée. Livestock Research for Rural Development. Vol 17 Art \# 34 Retrieved from

http://www.lrrd.org/lrrd17/3/tedo17030.h tm.

Ramirez J, Posada OS, Noguera R. 2015. Effects of Kikuyu grass (Pennisetum clandestinum) age and different forage: concentrate ratios on methanogenesis. Revista MVZ Cordoba vol. 20 no. 3; 12 p.

Ruggia CP, Kozloski GV, Bonnecarrère SLM, Lima LD, Oliveira L, Härter CJ,
Fiorentini G, Cadorin RL. 2008. Age of regrowth as a factor affecting the nutritive value of hay of kikuyu grass (Pennisetum clandestinum) offered to lambs. Grass Forage Sci., 63: 193-201. http://doi.org/10.1111/j.13652494.2007.00624.x

Suttie JM. 2004. Conservation du foin et de la paille: pour les petits paysans et les pasteurs. Collection FAO $\mathrm{n}^{\circ} 29 /$ Production végétale et protection des plantes. $301 \mathrm{p}$.

Tendonkeng F, Boukila B, Pamo TE, Mboko AV, Matumuini NEF, Zogang BF. 2009. Effet de différents niveaux de fertilisation azotée et du stade phénologique sur la croissance et le rendement de Brachiaria ruziziensis dans l'Ouest-Cameroun. Int. J. Biol. Chem. Sci., 3(4): 725-735. DOI: 10.4314/ijbcs.v3i4.47182

Tshibangu ML, Kampempa MF, Kashala KC, Kiatoko MH, Hornick JL. 2014. Composition chimique et indice de palatabilité des feuilles de Adenodolichos rhomboideus, Leucaena leucocephala et Stylosanthes guianensis chez la chèvre locale à Lubumbashi. Int. J. Biol. Chem. Sci., 8(3): 937-945. DOI: http://dx.doi.org/10.4314/ijbcs.v8i3.10

Van Soest PJ, Robertson JB, Lewis BA. 1991. Methods for dietary fibre, neutral detergent fibre and non-starch polysaccharides in relation to animal nutrition. J. Dairy Sci., 74(10): 35833597. DOI: $10.3168 /$ jds.S0022-0302 (91)78551-2

Zoffoun AG, Aboh AB, Adjolohoun S, Houinato M, Sinsin B. 2013. Effet de l'âge et de l'intensité de pâture sur le développement des touffes et la production de biomasse de Panicum maximum var. $\mathrm{C} 1$ dans les pâturages artificiels en zone soudanienne et subéquatoriale. Int. J. Biol. Chem. Sci., 7(3): 1168-1179. DOI : http://dx.doi.org/10.4314/ijbcs.v7i3.23 